\title{
Submitted Abstracts for Presentation at the AAPdN Annual Conference April 15-17, 2016 Flamingo Resort in Las Vegas, NV
}

Received: 29 April 2016 / Published online: 19 July 2016

(C) American Academy of Pediatric Neuropsychology 2016

Authors: Noah K. Kaufman, Ph.D. ${ }^{1}$ (noahk@ zianet.com), Yesenia Aguilar, M.S.W. ${ }^{1}$ (yesenia1@zianet.com), and Virginia Martinez, B.S.1 (iolkos@yahoo.com)

${ }^{1}$ Address: PO Box 16198, Las Cruces, NM 88004

Office Phone: 575-526-9090

Office Fax: 575-526-8787

Affiliation: Center for Neuropsychological Studies

\section{Neuropsychology Is Medically Necessary for Medicaid Youth in New Mexico}

\section{Objective}

The motivation for this commentary is to address a relatively straightforward, but important, question: Do children receiving Medicaid in New Mexico (NM) need neuropsychological testing? Are these healthcare services justified from a socioeconomic (direct and indirect costs to the community), ethical, or financial (costs to the managed care organizations [MCOs]) vantage point? Our assertion is that glaring neuropsychological deficits among Medicaid youth exist but that these deficits demand more scientific attention so that the medical necessity of neuropsychological testing can be demonstrated to Medicaid MCOs, to legislators, and to the consumers of these valuable services.

\section{Conclusions}

The official nickname for NM is the "Land of Enchantment" even though local statistics suggest a land of disenchantment: NM has the highest rate of unintentional drug overdose deaths in the nation ${ }^{1}$; NM leads the country in alcohol-related deaths ${ }^{2}$; NM is the second most violent state ${ }^{3}$ NM is the second poorest state $^{3}$; $\mathrm{NM}$ is the sixth most uneducated state ${ }^{3}$; and, since 2004, the number of NM youths overdosing from heroin has drastically spiked ${ }^{4}$. Not surprisingly, Medicaid-i.e., healthcare for poor children, pregnant women, people with disabilities, and other limited-asset members of society-is relied upon by over 700,000 citizens of $\mathrm{NM}^{5}$. This means almost $35 \%$ of the state qualifies for and receives this joint state-federal support.

We know that neuropsychological health is a good predictor of socioeconomic success ${ }^{6}$ as well as navigation through complex real-world environments ${ }^{7}$. Healthy brains tend not only to be more successful socioeconomically, but they are generally better at thinking their way through challenging real-world situations. It follows, therefore, that poorer states like NM might be populated by children with less neuropsychological health, thereby adding justification for neuropsychological testing among these youngsters.

\section{References}

New Mexico Department of Health: Substance Abuse Epidemiology Program IabEB, Epidemiology and Response Division, New Mexico Department of Health. Substance Abuse Epidemiology Program, Injury and Behavioral Epidemiology Bureau, Epidemiology and Response Division, New Mexico Department of Health. 2011; http://www.health.state.nm.us/erd/HealthData/ SubstanceAbuse/2010New MexicoSubstance Abuse Epidemiology Profile.pdf.

Malone P. New Mexico leads nation in alcohol-related deaths 2015; http://www.santafenewmexican.com/news/local news/newmexico-leads-nation-in-alcohol-related-deaths/article 410cab47-b75f-5210-b791-8ecalcfda0a4.html.

Kent A, Frohlich TC. The most dangerous states in America 2014; http://247wallst.com/special-report/2015/01/02/the-mostdangerous-states-in-america-2/4/. 
Shah N. Drug abuse patterns and trends in Albuquerque and New Mexico — update: January 2011.

Epidemiologic Trends in Drug Abuse, Proceedings of the Community Epidemiology Work Group, Highlights and Executive Summary. 2011:58-61.

Services CfMM. 2015; http://www.medicaid.gov/medicaid-chipprogram-information/by-state/new-mexico.html.

Strenze T. Intelligence and socioeconomic success: a metaanalytic review of longitudinal research. Intelligence. 2007;35(5):401-426.

Gottfredson L. Why g matters: the complexity of everyday life. Intelligence.//1997;24:79-132.

Authors: Noah K. Kaufman, Ph.D.' (noahk@zianet.com), Yesenia Aguilar, M.S.W. ${ }^{1}$ (yesenia1@zianet.com), and Virginia Martinez, B.S. ${ }^{1}$ (iolkos@yahoo.com)

\section{${ }^{1}$ Address: PO Box 16198, Las Cruces, NM 88004 \\ Office Phone: 575-526-9090 \\ Office Fax: 575-526-8787 \\ Affiliation: Center for Neuropsychological Studies}

\section{Smaller Finger Length Ratios in Unintended Pregnancies}

\section{Objectives}

The ratio of the index finger to the ring finger length $(2 \mathrm{D}: 4 \mathrm{D})^{1}$ reflects androgen exposure in utero (smaller ratios mean more androgen exposure); it correlates with in utero brain development ${ }^{1-3}$; it is relatively easy to measure; and it may add valuable information to pediatric neuropsychological workups. We compared 2D:4D ratios in those resulting from intended versus unintended pregnancies.

\section{Method}

We computed 2D:4D ratios, first in a youth sample ( $m$ age $=10.88$ years; $s_{\mathrm{age}}=3.73$ ) and then in a combined youth-adult sample $\left(m_{\mathrm{age}}=30.60\right.$ years; $\left.\mathrm{sd}_{\mathrm{age}}=23.40\right)$ to increase sample size $\left(N_{\text {youth }}=23 ; N_{\text {combined }}=55\right)$. We ran independent-samples $T$ tests for both hands, using 2D:4D ratios and pregnancy status (intended vs. unintended).

\section{Results}

Youth sample 2D:4D ratios were smaller in the unintended pregnancy group: $2 \mathrm{D}: 4 \mathrm{D}_{\text {right }}(m=.924 ; \mathrm{sd}=.039) ; 2 \mathrm{D}: 4 \mathrm{D}_{\text {left }}$ $(m=.924 ; \mathrm{sd}=.034)$. In contrast, youths from an intended pregnancy had larger ratios: $2 \mathrm{D}: 4 \mathrm{D}_{\text {right }}(m=.952$; $\mathrm{sd}=.0516) ; 2 \mathrm{D}: 4 \mathrm{D}_{\text {left }}(m=.952 ; \mathrm{sd}=.020)$. Combined youth-adult sample ratios remained smaller in the unintended pregnancy group (2D:4D $\mathrm{D}_{\text {right }}(m=.946 ; \mathrm{sd}=.044) ; 2 \mathrm{D}: 4 \mathrm{D}_{\text {left }}$ $(m=.950 ; \mathrm{sd}=.039))$, while the intended pregnancy ratios were larger: $2 \mathrm{D}: 4 \mathrm{D}_{\text {right }}(M=.964 ; \mathrm{SD}=.040) ; 2 \mathrm{D}: 4 \mathrm{D}_{\text {left }}$ $(m=.963 ; \mathrm{sd}=.024)$. Though not statistically significant (2D:4D right $t[36]=1.19, p=.241, d=.41 ; 2 \mathrm{D}: 4 \mathrm{D}_{\text {left }} t$ $[35]=1.60, p=.119, d=.50)$, these preliminary results say unintended pregnancies correspond with smaller 2D:4D ratios.

\section{Conclusions}

Unintended pregnancies are less healthy ${ }^{4}$, costly to society 5, 6 , and may disproportionally yield citizens who struggle to navigate real-world challenges ${ }^{7-9}$. Our findings say unintended pregnancies result in more in utero androgen exposure (i.e., smaller 2D:4D ratios) ${ }^{10,11}$, which might help explain this developmental sequence of events.

\section{References}

Manning JT. Digit ratio: a pointer to fertility, behavior, and health: Rutgers University Press; 2002.

Liu J, Portnoy J, Raine A. Association between a marker for prenatal testosterone exposure and externalizing behavior problems in children. Dev Psychopathol. Aug 2012;24(3): 771-782.

Lange BP. Digit ratio as a predictor of language development and media preferences in kindergarten children. Acta Linguistica. 2015;9(2).

Delgado-Rodríguez M, Gómez-Olmedo M, Bueno-Cavanillas A, Gálvez-Vargas R. Unplanned pregnancy as a major determinant in inadequate use of prenatal care. Preventive Medicine. 1997;26(6):834-838.

Sonfield A, Kost K, Gold RB, Finer LB. The public costs of births resulting from unintended pregnancies: national and state-level estimates. Perspectives on Sexual and Reproductive Health. 2011;43(2):94-102.

Monea E, Thomas A. Unintended pregnancy and taxpayer spending. Perspectives on Sexual and Reproductive Health. 2011;43(2):88-93.

Deary IJ, Strand S, Smith P, Fernandes C. Intelligence and educational achievement. Intelligence.//2007;35:13-21.

Strenze T. Intelligence and socioeconomic success: a meta-analytic review of longitudinal research. Intelligence. 2007;35(5):401426.

Gottfredson L. Why g matters: the complexity of everyday life. Intelligence.//1997;24:79-132.

Geschwind N, Galaburda AM. Cerebral lateralization: biological mechanisms, associations, and pathology: I. A hypothesis and a program for research. Archives of neurology. 1985;42(5):428.

Nguyen T-V, McCracken J, Ducharme S, et al. Testosteronerelated cortical maturation across childhood and adolescence. Cerebral cortex. 2013;23(6):1424-1432. 
Authors: Andrew S. Davis, Ph.D. ${ }^{1}$ (davis@bsu.edu), Noah K. Kaufman² (noahk@zianet.com), Marlyssa R. Fillmore, M.A. ${ }^{1}$ (mrroark@bsu.edu), D. J. Bernat, M.S.' (dbernat@bsu.edu), and Kelly L. Fogle, M.A. ${ }^{1}$ (klfogle@bsu.edu)

${ }^{1}$ Address: 2000 W. University Ave., Teachers College 505, Muncie, IN 47306

Office Phone: 765-285-8500

Office Fax: 765-285-3653

Affiliation: Ball State University

${ }^{2}$ Address: PO Box 16198, Las Cruces, NM 88004

Office Phone: 575-526-9090

Office Fax: 575-526-8787

Affiliation: Center for Neuropsychological Studies

\section{D:4D Finger Ratio and Parental Conduct Ratings: a Preliminary Analysis}

\section{Objectives}

Prenatal development variables have the potential to result in persistent physical dysmorphology, including craniofacial and hand/finger aberrations. Pediatric neuropsychologists should be aware of the implications suggested by physical dysmorphology, as physical features are more objectively quantifiable than associated neurocognitive deficits. This proposal reports the results of a preliminary analysis evaluating the relationship between the ratio of second and fourth digit length (2D:4D) and a parental objective rating form of conduct disturbance.

\section{Method}

Participants were 15 children (10 male, 5 female) referred for outpatient neuropsychological assessments ( $M$ age $=11.81$ years; $\mathrm{SD}=4.46)$. Diagnoses following assessment were mixed, and a full description will be included in the poster. Participants' parents completed the Conduct Disorder Scale (CDS) along with a measurement of left and right second (index) and fourth (ring) fingers in millimeters ( $\mathrm{mm}$ ) as part of a comprehensive neuropsychological evaluation.

\section{Results}

The mean left hand $2 \mathrm{D}: 4 \mathrm{D}$ ratio was $.94 \mathrm{~mm}$ $(\mathrm{SD}=.04 \mathrm{~mm})$, and the mean right hand $2 \mathrm{D}: 4 \mathrm{D}$ ratio was $.94 \mathrm{~mm}(\mathrm{SD}=.04 \mathrm{~mm})$. Mean $z$ scores on the CDS ranged from -1.56 to -2.04 with a mean Conduct Disorder Quotient of -2.28. Correlations between 2D:4D and scores from the CDS ranged from .013 to .32 with largely negative correlations. The largest correlation was seen between both left $(-.32)$ and right (-.32) 2D:4D with the Deceitfulness/Theft scale.

\section{Conclusions}

Our findings were consistent with the literature noting an inverse relationship between 2D:4D ratio and aggressive behavior in children, although results were not statistically significant. With a larger sample size, statistically significant correlations could emerge. This preliminary study suggests this line of research should continue. The poster will discuss implications for practitioners and researchers as well as future plans to continue this study.

Authors: Brenda Kepler Leske, Psy.D. ${ }^{1}$ (drleske@sbcglobal.net) and Cortney Bindrich, Ed.D.' (dr.bindrich@gmail.com)

Address: 4785 Hayes Road, Suite 100, Madison, WI 53704

Office Phone: ${ }^{1} 608-242-7160$ x226; ${ }^{2} 608-242-7160 \times 255$

Office Fax: 608-242-7153

Affiliation: Counseling Resources Neuropsychological Associates, LLC.

\section{"Arrested Development": Poor Psychosocial Outcome Despite Adequate Neuropsychological Profile with Anterior Left Hemisphere Dysfunction}

\section{Objective}

The aim of this case study is to review the complex and remarkable profile of a female adolescent patient 7 years post-brain tumor resection and at the time of her transition into post-secondary education. Despite her strong neuropsychological profile, she has been unsuccessful in transferring her abilities into everyday life.

\section{Method}

Patient $\mathrm{X}$ is an 18-year-old Caucasian female currently residing in the Midwest. She was diagnosed with a glioblastoma multiforme (GBM) in her left frontal/ temporal lobe and underwent resection of the tumor in the summer of 2009. She was administered a comprehensive battery of tests assessing general intelligence, reasoning, memory, motor skills, attention and executive functioning, academic achievement, auditory processing, and objective/projective personality measures. 


\section{Results}

Patient X's results from the neuropsychological evaluation revealed average to above average abilities across the majority of evaluation. To simply look at her scores from the evaluation, one would conclude that she possesses few (if any) deficits. However, she has been unable to functionally and emotionally transition into adulthood, lacking insight, task initiation, organization and planning, confabulation, and independent finance management. She has additionally withdrawn from college after her first semester due to aforementioned difficulties. Despite demonstrating strong cognitive abilities and receiving extensive cognitive retraining, patient $X$ is struggling to be independent against the effects of an absent anterior cingulate and frontal lobe.

Author: Cortney Bindrich, Ed.D. (dr.bindrich@gmail.com)

Address: 4785 Hayes Road, Suite 100, Madison, WI 53704

Office Phone: 608-242-7160 x225

Office Fax: 608-242-7153

Affiliation: Counseling Resources Neuropsychological Associates, LLC.

\section{Understanding and Consulting on the Neuropsychological Profile of Septo-Optic Dysplasia: a Case Study}

\section{Objective}

Parents will often refer their child to a pediatric neuropsychologist for an evaluation, hoping for a differential diagnosis. The aim of this case study is to discuss the complex and rare neuropsychological profile of a young boy whose parents were seeking an initial diagnosis of their child due to emotional, behavioral, and learning concerns. By compiling and integrating medical records, along with a parent interview, research articles, and the results of the neuropsychological battery, the translation of the diagnosis provided the patient's parent a new perspective and understanding into their child's behaviors.

\section{Method}

The patient is a 9-year-old Caucasian male currently residing in the Midwest. At 17 months of age, an MRI of the patient revealed a "dysplastic corpus callosum with clivus and sphenoid bowing more horizontally oriented than normally expected." There was also mild anterior kinking seen in the medulla near that level of the foramen magnum. Observations of patient $X$ included short stature, significant deficits in attention and concentration, and verbal perseverations. He was administered a comprehensive battery of tests assessing general intelligence, reasoning, language, memory, motor skills, attention and executive functioning, auditory processing, and objective/projective personality measures.

\section{Results}

The neuropsychological evaluation results, along with supporting developmental history and medical records, revealed a profile of strengths and weaknesses most similar to individuals with septo-optic dysplasia (SOD), including impaired intellectual functioning, along with deficits in visualspatial integration, abstract reasoning, and cognitive flexibility. Upon receiving the results and discussing the neurocognitive, behavioral, social, and adaptive profile of individuals with SOD with the clinician, patient X's parents were able to gain new insights into their child. This lead to specific interventions in his school and with his therapist that were tailed to his unique learning, behavioral, and emotional needs.

Authors: Meghan N. Cahill, M.A.1 (Meghan.cahill@my.wheaton.edu), Peter Dodzik, Psy.D., ABPdN, ABN², Arina Polevoy, Psy.D. ${ }^{2}$, Benjamin Pyykkonen, Ph.D. ${ }^{1}$ (ben.pyykkonen@wheaton.edu), Ewelina Lakomy $^{2}$, and Kelsey Oster, M.A. ${ }^{2}$

${ }^{1}$ Address: 12501 College Ave., Wheaton, IL 60187

Phone: (815) 603-5058

Fax: (630) 752-7033

Affiliation: Wheaton College

${ }^{2}$ Address: 121 S. Wilke Ave, Suite 200, Arlington Heights, IL 60005

Phone: (847) 577-0904

Fax: (847) 577-1558

Email: lcamp@nwbhs.com

Affiliation: Northwest Behavioral Health Services

Using the D-KEFS Tower Test to Examine ADHD Sensitivity in Children: Expanding Analysis Beyond the Summary Score

\section{Objectives}

The objective of this study was to evaluate the clinical utility of the Delis-Kaplan Executive Function Systems (D-KEFS) Tower Test in the assessment of ADHD. Specifically, the study aimed to evaluate the utility of 
the Optional Measures from the Tower for diagnostic accuracy as well as correlation with several subjective and objective instruments commonly used in the assessment of ADHD.

\section{Methods}

An archival analysis was performed on 118 subjects (mean full scale IQ $=106.5, \mathrm{SD}=13.67$; mean age $=14.08, \mathrm{SD}=2.83 ; 48$ female). Ninety-eight subjects had a primary diagnosis of ADHD, and 20 were diagnosed with mood, LD, or received no diagnosis. All subjects completed the D-KEFS Tower as part of a more broad neuropsychological evaluation. Most subjects completed the Behavior Assessment Scale for Children 2, the Behavioral Rating Inventory of Executive Function, and the Conners' Continuous Performance Test 2.

\section{Results}

Subjects with ADHD were no more likely than normal or clinical controls to show impairment on the Total Achievement Score, which is consistent with previous literature. However, subjects with ADHD were twice as likely to score above 1 standard deviation on mean first-move time $\left(X^{2}=[1, N=117] 6.44, p=0.01\right)$. Subjects with ADHD were over three times more likely to fall 1 standard deviation below the mean on mean accuracy ratio $\left(X^{2}=[1, N=117]=24.82\right.$, $p>0.001)$. Lower scores on several Tower Optional Measures were highly predictive of parent-completed rating scales and the CPT-2.

\section{Conclusions}

Results from this study indicate that the Optional Measures of the D-KEFS Tower are more useful in the assessment of ADHD than the Total Achievement Score. Additional analyses will be included.

Authors: Stacy Watson, MA, LCPC ${ }^{1}$ (swatson@harpercollege.edu), Michele DeCanio, MA, LCPC ${ }^{1}$, Peter Dodzik, PsyD, ABPdN, $\mathrm{ABN}^{2}$, Ewelina Lakomy, $\mathrm{BA}^{2}$, Taylor Hughes, $\mathrm{BA}^{2}$, and Kelsey Oster, $\mathrm{MA}^{2}$

${ }^{1}$ Address: 1200 W. Algonquin Road, Palatine, IL 60067

Phone: (847) 925-6266

Affiliation: Harper College

${ }^{2}$ Address: 121 S. Wilke Ave, Suite 200, Arlington Heights, IL 60005

Phone: (847) 577-0904

Fax: (847) 577-1558

Email: lcampo@nwbhs.com
Affiliation: Northwest Behavioral Health Services

Evaluation of a Pilot Program for the Support of College Students with Autism Spectrum Disorders: Project TAP (Transition Autism Program)

\section{Objectives}

The aim of this study was to evaluate a novel support services program for entering college students with autism spectrum disorder (ASD).

\section{Methods}

Prospective data was collected on students with ASD enrolling at a Midwest college. Thirty-four students with ASD volunteered to participate in this study. Sixteen (75\% Caucasian) students were enrolled in the Transition Autism Program (TAP) which provides evidence-based peer and disability support. The remaining ASD students $(n=18,89 \%$ Caucasian) were enrolled as students with disabilities but did not participate in the program. All subjects completed the Student Adaptation to College Questionnaire (SACQ) as the primary measure of social and emotional adjustment to college. Information regarding the students' attrition, GPA, and course completion were also analyzed.

\section{Results}

ASD students in the TAP program $(\mathrm{GPA}=3.42$, $\mathrm{SD}=1.14)$ demonstrated significantly higher GPA than non-TAP ASD students $(\mathrm{GPA}=2.50, \mathrm{SD}=0.58 ; t=2.85$, $p=0.025)$. In addition, students in the TAP program were significantly less likely to be on academic probation than their non-TAP ASD counterparts $\left(X^{2}[1, n=34]=6.11\right.$, $p=0.013$ ). No group differences were found between level of academic, social, personal, emotional, or attachment scales from the SACQ at the beginning of the study. TAP students showed average adjustment on primary measures of SACQ at the beginning and end of the study.

\section{Conclusions}

Results from this pilot study indicate that participation in the TAP program by ASD students resulted in significantly higher overall GPA, reduced academic probation, and lower attrition rates when compared to other ASD students, other students with disabilities, and general education students. No significant differences on measures of global academic adjustment were found. This study demonstrates that proactive support for ASD students may result in significant improvement in college performance and retention. 
Author: Ludmila Zaytsev, Ph.D. (ludmila.zaytsev@cshs.org)

Address: 19510 Ventura Blvd., Ste. 202, Tarzana, CA 91356 Phone: 310-423-9884

Fax: 310-423-9222

Affiliation: Cedars-Sinai Health Systems, Dept. of Physical Medicine and Rehabilitation

\section{Cognitive Sequelae of Nephronophthisis 4: a Sibling Case Study}

\section{Objective}

Nephronophthisis (NPHP), an autosomal recessive cystic kidney disease with positional cloning on nine genes (NPHP1-NPHP9), is the most frequent genetic cause for end-stage renal disease (ESRD) in children and adolescents. It is associated with multiple organ involvement. Neuropsychological outcomes literature is largely limited to NPHP1 studies. Identified areas of deficit are visuomotor processing, verbal memory, impulsivity, motor coordination, and cognition. This sibling study offers information regarding sequelae of NPHP4 in the context of prenatal poly-substance exposure.

\section{Method}

Case study of two siblings with NPHP4 mutation was confirmed by molecular testing. Sibling 1 was a 17year-old male, post-1 year of dialysis, and had undergone renal transplant at age 10 . Sibling 2 was an 18year-old female, diagnosed with progressive chronic $\mathrm{RD}$, and had post-preemptive renal transplant at age 15. Presenting complaints include executive dysfunction and mood disturbance. Both underwent comprehensive neuropsychological evaluations.

\section{Results}

Both siblings evidence lateralized neuropsychological profile with average verbal skills and compromised visual processes. Receptive and expressive language skills, verbal fluency, verbal memory, and sustained attention are intact. Divided attention, visual memory, visual-motor integration, and fine motor dexterity are deficient. Suboptimal processing speed and selfmonitoring are sibling 1 specific.

\section{Conclusions}

There are distinct differences in the neuropsychological outcomes of NPHP1 and NPHP4. After the effects of polysubstance exposure are accounted, NPHP4 has a lateralized profile suggesting right occipito-parietal involvement; dorsolateral prefrontal cortex, subcortical structures, and cerebellum are also implicated. Outcome differences between siblings might be related to the disease stage at the diagnosis, followup medical compliance, and/or sex protective factors.

Authors: Jason Swift, M.S. (swif7572@pacificu.edu) and Susan T. Li, Ph.D. (susanli@ pacificu.edu)

Address: 190 SE 8th Avenue, Suite 260, Hillsboro, OR 97123 Phone: 503-352-2610

Fax: 503-352-7320

Effects of Anxiety Symptoms and Gender on Neuropsychological Test Performance in Children with Brain Tumor

\section{Objective}

Anxiety is one of the most prevalent affective disturbances associated with pediatric cancer (Turner et al. 2009) with girls reporting higher levels than boys (Maurice-Stam et al. 2008). The purpose of this study is to investigate the differential effects of anxiety on neurocognitive functioning in girls and boys diagnosed with brain tumor (BT). Given previous findings (Moitra and Armstrong, 2013), we hypothesized that girls would have significantly more anxiety than boys and consequently larger neurocognitive deficits.

\section{Method}

Participants included $24(50 \%$ male) pediatric patients diagnosed with BT ages 6-16 who underwent neuropsychological evaluation. Data were collected from evaluations conducted as part of routine care through a survivorship clinic at a medical university. Pearson correlations and regression analyses were conducted to determine the extent to which reported anxiety (RCMAS) was associated with performance on measures of mental set shifting (WCST) and processing speed (Trails A).

\section{Results}

There was no difference between boys' $(M=45.7)$ and girls' $(M=39.8)$ reported anxiety $(t=1.65, p>.05)$; thus, the entire sample was analyzed together. Anxiety was unrelated to processing speed (all $p \mathrm{~s}>.05$ ). There was a positive correlation between anxiety and failure to maintain set $(r=.53, p=.015)$. Anxiety positively predicted failure to maintain set $(\beta=.53, p=.015, \mathrm{CI}=0.1$ to 0.9$)$ and explained $29 \%\left(R^{2}=.53\right)$ of the variance in performance. 


\section{Conclusions}

Unexpectedly, boys and girls with BT did not differ in anxiety levels. Among children with BT, anxiety was associated with decreased ability to maintain mental set, but processing speed was unrelated. Anxiety, whether a direct result of BT or a response to diagnosis and subsequent cancer treatment, may influence neuropsychological performance.

Authors: Christopher J. Nicholls, Ph.D., ABPdN, ABPP ${ }^{1}$ (cn@thenichollsgroup.com), Emily Ross, M.A. ${ }^{1}$ (er@thenichollsgroup.com), and W. Joel Schneider, Ph.D. ${ }^{2}$ (wjschne@ilstu.edu)

${ }^{1}$ Address: The Nicholls Group, 9965 N. 95th Street, Suite 101, Scottsdale, AZ 85258

Phone: 480-998-2303

Fax: 480-998-3169

${ }^{2}$ Address: Illinois State University, Psychology Department, 447 DeGarmo, Campus Box 4620, Normal IL 61790-4620

Phone: 309-438-8410

\section{The Neuropsychological Profiles of Twins Discordant for} Sotos Syndrome: a Case Study

\section{Objective}

Sotos syndrome is a genetic syndrome that has neuroimaging anomalies including large ventricles, increased extracerebral fluid, and midline abnormalities. ${ }^{1}$ Other findings implicate hypoperfusion of frontal brain areas. ${ }^{2}$ The current report compares the neuropsychological profiles of fraternal twins discordant for Sotos syndrome.

\section{Method}

A neuropsychological evaluation of the 7-year-old, second grade, affected proband (twin A) was compared with a similar evaluation performed upon his brother (twin B).

\section{Results}

Twin A's (Sotos) neuropsychological profile was characterized by impaired spatial reasoning and working memory, as compared with relatively more preserved verbal and nonverbal reasoning skills. Performance on overlearned and concrete information was superior to novel conceptual learning and global (as opposed to local) information processing. Mathematics problem solving was more impaired than early reading skills. Frontal/executive functions were significantly impaired. Adaptive behavioral skill development was less impaired, falling in the low average range overall. Comparison of twins $\mathrm{A}$ and $\mathrm{B}$ documented significant differences in all areas except fine motor speed, with largest differences observed in dorsal stream visuospatial/constructional functions and working memory. Social perception and complex fine motor coordination were impaired in proband but not brother.

\section{Discussion/Conclusion}

The current findings are consistent with previous reports that Sotos syndrome may present with evidence of a mixed right hemisphere learning disability and a profile of executive function impairments. These findings point to the necessity of evaluating individuals with Sotos syndrome at a neuropsychological level, as reliance upon more global intelligence test scores inadequately describes their abilities.

\section{References}

Schaefer, GB, Bodensteiner JB, Buehler, BA, et al. (1997) The neuroimaging findings in Sotos syndrome. AM J Med Genet: 68: 462-465.

Horikoshi, H., Kato, Z, Masuno, M et al. (2006) Neuroradiologic findings in Sotos syndrome. Journal of Child Neurology 21:7 614-618.

Authors: Andrew S. Davis, Ph.D. ${ }^{1}$ (davis@bsu.edu), Noah K. Kaufman, Ph.D. ${ }^{2}$ (noahk@zianet.com) Marlyssa Fillmore, MA ${ }^{1}$ (mrroark@bsu.edu), D.J. Bernat, MS ${ }^{1}$ (dbernat@bsu.edu), Brittney Moore, MA ${ }^{1}$ (bmklauser@bsu.edu), and Virginia Martinez, BS ${ }^{2}$ (iolkos@yahoo.com)

${ }^{1}$ Address: 2000 W. University Ave., Teachers College 505, Muncie, IN 47306

Office Phone: 765-285-8500

Office Fax: 765-285-3653

Affiliation: Ball State University

${ }^{2}$ Address: PO Box 16198, Las Cruces, NM 88004

Office Phone: 575-526-9090

Office Fax: 575-526-8787

Affiliation: Center for Neuropsychological Studies

\section{Resting Heart Rate and Antisocial Behavior in a Multicultural Youth Sample}

\section{Objectives}

Neuropsychology practice is enhanced when biomarkers are integrated with traditional neuropsychological assessment 
measures. While a recent meta-analysis (Portnoy et al. 2015) conveyed an association between low resting heart rate and antisocial behavior, the relationship is not fully understood, particularly with ethnically diverse youth (Fletcher-Janzen et al. 2013). Our objective is to chart this territory in a multicultural youth sample, using parent reports of antisocial behavior and resting heart rate.

\section{Method}

Participants were 15 children referred for outpatient neuropsychological assessment (mean age $=11.81$ years; $\mathrm{SD}=4.46 ; 13 \%$ Caucasian). Multiple diagnoses were made following assessment, including ADHD and mild intellectual disability (ID); a full description will be included in the poster. Participants' parents completed the Conduct Disorder Scale (CDS), while readings of resting heart rate among child examinees were taken as part of a comprehensive neuropsychological evaluation.

\section{Results}

Mean resting heart rate was 77.47 beats per minute (bpm; $\mathrm{SD}=13.79$ ), and mean $z$ scores on CDS composite scales ranged from -1.55 to -2.04 . Correlations between resting heart rate and $z$ scores from the CDS were all positive: $r=.376$ at the low end and $r=.576$ at the upper end. A regression model revealed resting heart rate predicted a substantial proportion of the variance in the Hostility CDS composite scale $\left(R^{2}=.331 ; p=.025\right)$.

\section{Conclusions}

Our findings strongly diverge from the existing literature, which emphasizes a link between low resting heart rate and antisocial behavior. Hence, demographic factors including ethnic background may moderate the relationship between resting heart rate and antisocial behavior. This is a report of preliminary findings, and the poster will discuss implications for practitioners and researchers, along with future plans to continue this study.

Authors: Andrew S. Davis, Ph.D. (davis@bsu.edu), Stephanie L. Simon-Dack, Ph.D. (slsimondack@bsu.edu), Kelly L. Fogle, M.A. (klfogle@bsu.edu), and Elizabeth R. Lemann, Ph.D. (elroberds@bsu.edu)

Address: 2000 W. University Ave., Teachers College 505, Muncie, IN 47306

Office Phone: 765-285-8500

Office Fax: 765-285-3653

Affiliation: Ball State University
QEEG Beta and Gamma Activation Associated with Inhibition

\section{Objectives}

Resting quantitative electroencephalography (QEEG) is an electrophysiological recording method that digitizes brain signals, so they can be analyzed mathematically. Electroencephalography is primarily used in clinical neurology and in psychology as a research tool; however, it is not frequently used in clinical neuropsychology. This study evaluated the relationship between a standardized and norm-referenced measure of executive functioning and electrophysiological activity.

\section{Method}

Participants were 41 college students (18 male, 23 female) with a mean age of $19.54(\mathrm{SD}=1.57)$. Participants completed a QEEG session and neuropsychological tasks as part of a research study for extra credit in an undergraduate psychology course. The relationship between the Inhibition/Switching test from the Delis-Kaplan Tests of Executive Functioning (DKEFS) and baseline EEG frequencies recorded at rest with eyes open was studied. Predictor variables were alpha, beta, and gamma at the right and left frontal and parietal sites.

\section{Results}

$\mathrm{n}$ average, $131 \mathrm{~s}$ of artifact-free data was obtained after artifact rejection. The mean Inhibition/Switching error score was 11.22 with a standard deviation of 1.35 . Regression analysis indicated the QEEG measurements predicted a significant proportion of the variance in Inhibition/Switching error scores $\left(R^{2}=.517 ; p=.022\right)$. The most significant predictor site was gamma left parietal.

\section{Conclusions}

The results suggested decreased levels of resting gamma activity in the left parietal lobe predicted better performance (fewer errors) on the Inhibition/Switching task. Right parietal gamma and left and right frontal beta activity approached significance. Research has consistently indicated a diffuse pattern of neurological activity in executive functioning, including frontal and parietal areas. Results and implications for practitioners and researchers will be discussed.

Authors: James Trinkle, Ph.D. (jtrinkle@blackfordschools.org), Andrew S. Davis, Ph.D. (davis@bsu.edu), W. H. Finch, Ph.D. (whfinch@bsu.edu), George Gaither, Ph.D. (ggaither@bsu.ed), D.J. Bernat, MS (dbernat@bsu.edu), and Marlyssa Fillmore, MA (mrroark@bsu.edu) 
Address: 2000 W. University Ave., Teachers College 505, Muncie, IN 47306

Office Phone: 765-285-8500

Office Fax: 765-285-3653

Affiliation: Ball State University

\section{Predicting High-Stakes State Test Results with CHC} Factors

\section{Objectives}

High-stakes state test results are coming under increasing scrutiny as they are increasingly used for educational and financial decisions, at both the school and state level. However, little research has been conducted into which neuropsychological factors are predictive of these test results for children referred for special education. This study investigated the relationship between Indiana Statewide Testing for Educational Progress-Plus (ISTEP+) scores and Cattell-Horn-Carroll (CHC) factors.

\section{Method}

Participants were 40 (mean age $=11.1$ years; $\mathrm{SD}=2.3$ years) students who completed the Woodcock-Johnston Tests of Cognitive Ability-Third Edition (WJ III-COG) and Woodcock-Johnson Tests of Academic Achievement (WJ III$\mathrm{ACH})$ as part of a psychoeducational evaluation. All participants were referred for an assessment to determine eligibility for special education assessment.

\section{Results}

Regression analysis indicated that the seven subtests of the WJ III-COG were not significantly related to the English/ Language Arts score of the ISTEP+ $\left(R^{2}=.199, F(7\right.$, $33)=1.174, p=.344)$ or to the Math score of the ISTEP+ $\left(R^{2}=.168, F(7,33)=.953, p=.480\right)$. Three reading subtests from the WJ III-ACH were related to the English/Language Arts score $(F(3,53)=2.959, p=.040)$ with the strongest relationship being found for passage comprehension. Three Math subtests from the WJ III-ACH were not related to the Math portion of the ISTEP+ $(F(3,53)=2.673, p=.057)$.

\section{Conclusions}

The results of this study raise some concerns for pediatric neuropsychologists and school psychologists who may be using CHC factors, particularly the cognitive scores, to provide recommendations for improving academic performance. It is also concerning there appears to be a disconnect between psychometrically sound measures of academic performance and high-stakes test. Implications for researchers and practitioners will be discussed.
Authors: Alison Colbert, M.A. ${ }^{1}$ (Acolber1@emich.edu) and Jin Bo, Ph.D. ${ }^{2}$ (Jbo@emich.edu)

${ }^{1}$ Address: 341 Science Complex, Ypsilanti, MI 48197

Office Phone: 720.777.6214

Office Fax: 734.487.6553

Affiliation: Eastern Michigan University

22Address: 301 M Science Complex, Ypsilanti, MI 48197

Office Phone: 734.487.3416

Office Fax: 734.487.6553

Affiliation: Eastern Michigan University

\section{Comparisons of Clinical and Experimental Working Memory Assessment Measures in Children}

\section{Objectives}

There are a number of methods for assessing working memory (WM), a limited-capacity system that has evolved for shortterm maintenance and manipulation of information. Span tasks are commonly utilized in clinical psychology, whereas change-detection tasks are often used in experimental psychology. This study sought to validate use of changedetection tasks in children, as well as evaluate the similarity between the change-detection tasks and clinical WM assessment.

\section{Methods}

Participants included 50 male children $(M=9.12$; range 612 years old) and 15 neurotypical male adults $(M=21.4$; range 19-28 years old). Child participants completed the twosubtest WASI-II followed by the WISC-IV and changedetection WM tasks. Administration of verbal and visual WM tasks was counterbalanced. Adult participants completed a demographic and screening form, WASI-II, and changedetection tasks.

\section{Results}

In the child sample, verbal working memory (VWM; $M=1.22, \mathrm{SD}=1.06)$ and visual spatial working memory (VSWM; $M=1.87, \mathrm{SD}=.96$ ) capacities were significantly smaller than adult capacity $(t=-5.13$ and -5.15 , respectively, both $p<.001)$. Visual inspections of WM capacity revealed a systematic decline in performance in both VWM and VSWM with increased set size. A trend for developmental increase in both VSWM and VWM was found $(r=.31$ and $r=.33$, respectively, $p<.05$ ), supporting significant age-related changes of WM in 
childhood. Although clinical WM measures were significantly correlated with each other $(r=.30-.85$, $p<.05-.01)$, only the Arithmetic subtest was significantly correlated with change-detection VSWM $(r=.34$, $p<.05)$, and none were significantly correlated with change-detection VWM.

\section{Conclusions}

Results suggest WISC-IV WM subtests do not consistently elicit the same construct as experimental measures, with the exception of the Arithmetic index. They also highlight the need for careful consideration of measure development, selection, and interpretation.

Author: Marlyssa R. Fillmore, M.A. (mrroark@bsu.edu)

Address: 2000 W. University Ave., Teachers College 505, Muncie, IN 47306

Office Phone: 765-285-8500

Office Fax: 765-285-3653

Affiliation: Ball State University

\section{Academic Performance in Students with Epilepsy: a Recent Review}

\section{Objectives}

The presence of academic difficulties in students with epilepsy has been well-established in the literature. What has been less well-established are the underlying variables associated with these difficulties. While some studies have largely attributed achievement problems to inherent cognitive difficulties in the population, other researchers have offered alternative explanations. Teacher attitudes, missed instruction, and individual adaptive competencies are all proposed sources of academic difficulty. As academic achievement is generally correlated with obtaining gainful employment (which is tied to overall life satisfaction), it is important to understand and address as many of these variables as possible. The purpose of this literature review is to examine potential factors associated with low academic achievement in students with epilepsy.

\section{Data Selection}

Literature on epilepsy and school achievement was obtained via searches of peer-reviewed journals available in the following databases: PsycINFO, PsycARTICLES, ERIC, MEDLINE, PubMed, and Academic Search Premier, using combinations of the following search terms: "epilepsy," “seizure," “achievement," “classroom," "integration," "missed instruction," and "academic." All relevant articles obtained were reviewed and synthesized.

\section{Data Analysis}

Studies obtained in the literature search were organized by proposed factors associated with academic performance. Measures of achievement were considered via these factors.

\section{Conclusions}

Based on the synthesis of the available literature, conference attendees will gain an understanding of the multifaceted nature of the issue of academic underachievement in students with epilepsy. Given the prevalence of epilepsy, associated academic difficulties, and long-term implications of academic performance, identification of underlying factors should be of interest to pediatric neuropsychologists to facilitate the design of strength-based interventions. Clinicians may better advise parents and schools of ways to minimize epilepsy's effect on academic achievement and improve resiliency.

Authors: Peter Dodzik, Psy.D., ABPdN, ABN ${ }^{1}$, Tasha Williams, Ph.D. ABPdN ${ }^{2}$ (twilliams@fwnc.com), Omar Sohail, B.S. ${ }^{2}$, Kelsey Oster, M.A. ${ }^{1}$, Erin Miles, Psy.D. ${ }^{2}$

${ }^{1}$ Address: 121 S. Wilke Ave, Suite 200, Arlington Heights, IL 60005

Phone: (847) 577-0904

Fax: (847) 577-1558

Email: lcampo@nwbhs.com

Affiliation: Northwest Behavioral Health Services

${ }^{2}$ Address: 11104 Parkview Circle, Suite 110, Fort Wayne, IN 46845

Phone: (260) 460-3100

Fax: (260) 460-3130

Affiliation: Fort Wayne Neurology

The Clinical Utility of the Gilliam Autism Rating Scale 3 (GARS-3) in the Assessment of Autism Spectrum Disorders

\section{Objectives}

The objective of this study was to evaluate the diagnostic accuracy of the Gilliam Autism Rating Scale 3 (GARS-3) compared to its predecessor in the assessment of autism spectrum disorders (ASD).

\section{Methods}

The authors conducted an archival and prospective analysis of 122 subjects with known ASD (female $=33$, age $=7.52$ $[\mathrm{SD}=3.62]$, full scale $\mathrm{IQ}=79.11[\mathrm{SD}=21.06])$ and 31 clinical controls (female $=7$, age $=8.94[\mathrm{SD}=3.83]$, full scale 
$\mathrm{IQ}=90.87[\mathrm{SD}=16.91])$. All subjects were administered either the GARS-2 or GARS-3 as part of their neuropsychological evaluation.

\section{Results}

There were no significant differences between the mean GARS-2 total score $(X=93.57, \mathrm{SD}=19.20)$ and the GARS3 total score $(X=95.50, \mathrm{SD}=15.51)$ for subjects with ASD. However, using the GARS-3 recommended cutoff score (total $>55$ ) resulted in all subjects (ASD and clinical controls) being classified as ASD. Using the recommended GARS-2 cutoff score (total $\geq 85$ ) yielded a PPP of 1.00 and negative predictive power (NPP) of 0.39 for the GARS-2 (sensitivity $=66 \%$, specificity $=100 \%$ ) and PPP of 0.72 and NPP of 0.00 for the GARS- 3 (sensitivity $=83 \%$, specificity $=46 \%$ ). Further analysis of correlations with other measures of ASD and GARS-3 profile analysis will be discussed.

\section{Conclusions}

Clinicians should take caution when using the GARS-3 recommended cutoff scores for ASD in clinical practice. Though the previous GARS-2 recommended cutoff score indicated slightly improved sensitivity, over half of the clinical controls were diagnosed with ASD.

Authors: Kelly L. Fogle, M.A. (klfogle@bsu.edu) and Stephanie L. Simon-Dack, Ph.D. (slsimondack@bsu.edu)

Address: 2000 W. University Ave., Teachers College 505, Muncie, IN 47306

Office Phone: 765-285-8500

Office Fax: 765-285-3653

Affiliation: Ball State University

\section{Cognitive Flexibility, Interhemispheric Transfer, and QEEG in Female Athletes}

\section{Objectives}

Sports-related concussions are known to cause neurocognitive and neurological deficits, and recent research suggests these problems last longer than previously anticipated. In this study, a Stroop task, a computerized interhemispheric transfer time (IHTT) task, and QEEG recordings were used to differentiate female athletes who had never sustained a concussion from those who sustained concussions in the past (i.e., at least 6 months prior to participating).

\section{Method}

Participants were 28 female athletes recruited through an introductory psychology subject pool, campus-wide emails, and other participants. Participants received course research credit or minimal monetary compensation. Fourteen participants with no concussion history comprised the control group
$(M$ age $=20.04)$, and 14 participants with a history of one or more concussions comprised the concussion group ( $M$ age $=20.40)$. All participants underwent QEEG recording and completed health and handedness surveys, a computerized IHTT task, and a Stroop task. Data were used to determine whether differences in neural firing, IHTT, and cognitive flexibility (i.e., task switching) could differentiate between participants in the control and concussion groups.

\section{Results}

Cognitive flexibility, IHTT, and four EEG frequencies were used as predictor variables in a discriminant function analysis. Although results were not statistically significant $(p>.05)$, the predictor variables accounted for $27.1 \%$ of the variance and correctly classified a sizable proportion of cases $(69.7 \%)$. Stroop switching task completion time and theta frequency power contributed most to group separation.

\section{Conclusions}

This study represents a unique contribution to the concussion literature because it focuses solely on female athletes and examines potential lasting concussion-related deficits. Although results were not statistically significant, differences in neural firing, IHTT, and Stroop performance explained a sizeable proportion of the variance between groups. Future research may expand current knowledge regarding lasting effects of concussion.

Authors: Brittney M. Moore, M.A. (bmklauser@bsu.edu), Angela M. Mion, M.A. (ammion@bsu.edu), Kelly Fogle, M.A. (klfogle@bsu.edu), Andrew S.Davis, Ph.D. (davis@bsu.edu), and Lisa Rubenstein, Ph.D. (lmrubenstein@bsu.edu)

Address: 2000 W. University Ave., Teachers College 505, Muncie, IN 47306

Office Phone: 765-285-8500

Office Fax: 765-285-3653

Affiliation: Ball State University

\section{Recent Advances in Neuropsychological Assessment to Facilitate Identifying Giftedness}

\section{Objectives}

Clinical neuropsychology is the practical application of the relationship between behavior and the central nervous system, as well as physiological and environmental factors that influence these variables. If there is an assumption that intellectual giftedness has a neurological component (e.g., intellectual ability is at least somewhat determined by brain functioning), then quantification of neurocognitive functioning should be considered a critical component of the process of identifying 
intellectual giftedness in children. The purpose of this poster is to discuss methods of giftedness identification by expanding current techniques to consider a wider array of neurocognitive abilities.

\section{Data Selection}

A review of literature was conducted by accessing the PsycINFO, PubMed, and Academic Search Premier databases. All searches were limited to peer-reviewed journal articles. Truncal search terms related to giftedness and intelligence, executive functioning, processing speed, attention, working memory, memory, and sensory-motor function were used. Articles were then selected based on their relevance to neuropsychological outcomes for gifted samples.

\section{Data Analysis}

Results of the relevant literature were organized by neuropsychological domain (i.e., executive functioning, processing speed, attention, working memory, memory, and sensory-motor function) and assessment measure in order to determine and compare the neuropsychological outcomes across gifted samples. Samples of studies with contradictory findings were evaluated to determine if inclusion criteria or criteria for being identified as gifted could explain outcome differences.

\section{Conclusions}

Based on the summary of the literature, participants will gain an understanding of neuropsychological assessment outcomes for a gifted sample in addition to how neuropsychological assessment can be considered in order to (1) assist in gifted identification of children and (2) identify possible educational performance outcomes for gifted students based on neuropsychological functioning.

Authors: Renee Allen, Ph.D. (reneeallenph.d@gmail.com), Angela M. Mion, M.A. (ammion@bsu.edu), Andrew S. Davis, Ph.D. (davis@bsu.edu), W. Holmes Finch, Ph.D. (whfinch@bsu.edu), Sharon Bowman, Ph.D. (sbowman@bsu.edu), and DJ Bernat M.S. (dbernat@bsu.edu)

Address: 2000 W. University Ave., Teachers College 505, Muncie, IN 47306

Office Phone: 765-285-8500

Office Fax: 765-285-3653

Affiliation: Ball State University

\section{The Relationship Between Executive Functioning, Intelligence, Stress, and Life Satisfaction}

\section{Objectives}

It has been suggested that certain executive functions are more related to intelligence than others (Friedman et al. 2006) including a stronger relationship for fluid intelligence than crystallized intelligence (Duncan et al. 1995). Life satisfaction is defined as an overall cognitive assessment of one's quality of life or state of affairs (Shin and Johnson 1978) and is an outcome measure often studied in relation to stress. The purpose of this study was to explore the interaction between mental flexibility, inhibition, and intellectual functioning and the ability of these neurocognitive functions to affect stress and life satisfaction.

\section{Methods}

Participants were 104 undergraduate participants with a mean age of 19.9 years. Participants completed the Wechsler Abbreviated Scale of Intelligence, DelisKaplan Executive Function System Trail-Making Test and Color-Word Interference Test, The Revised University Student Hassle Scale, and The Extended Satisfaction with Life Scale. Multiple structural equation models were fit to the data in order to evaluate the proposed models.

\section{Results}

Results of structural equation modeling indicated the primary model had the best fit and was structured with intelligence being the source variable that directly and indirectly affected life satisfaction through the partially mediating variables of inhibition and cognitive flexibility. In the primary model, stress was illustrated as directly affecting inhibition, cognitive flexibility, and life satisfaction.

\section{Conclusions}

These results suggest that executive functions are not independent of intellectual ability; executive functions and stress may be important factors in predicting life satisfaction. Findings suggest that intelligence is an important protective factor against a variety of negative outcomes, although other variables may be more important in predicting what Okun and Stock (1987) defined as one's current quality-of-life judgments, which are based on personal criteria for success and happiness.

Authors: Kelly L. Fogle, M.A. (klfogle@bsu.edu), Angela M. Mion, M.A. (ammion@bsu.edu), Brittney M. Moore, M.A. (bmklauser@bsu.edu), Andrew S. Davis, Ph.D. (davis@bsu.edu), and Lisa Rubenstein, Ph.D. (lmrubenstein@bsu.edu)

Address: 2000 W. University Ave., Teachers College 505, Muncie, IN 47306

Office Phone: 765-285-8500

Office Fax: 765-285-3653

Affiliation: Ball State University 
Neuroimaging for Identification of Giftedness: a Recent Review

\section{Objectives}

Clinical neuropsychology has traditionally emphasized the measurement of neurocognitive deficits rather than normative neurocognitive strengths; therefore, the field has minimally contributed to the gifted literature. Although quantitative measures of intelligence/achievement are frequently used to make decisions regarding eligibility for gifted education services, using these measures may introduce bias into the process because they do not necessarily measure the same constructs for minority or non-English speaking children as they do for children from the majority background. This is concerning as the demographic makeup of the USA becomes more diverse. Thus, this literature review examines potential alternative/ supplemental measures of neurological potential (i.e., how neuroimaging might inform identification of gifted students?).

\section{Data Selection}

Literature on giftedness and neuroimaging was obtained via searches of peer-reviewed journals available on Google Scholar, PubMed, and EBSCO Host in the following databases: PsycINFO, PsycARTICLES, ERIC, MEDLINE, and Academic Search Premier using combinations of the following search terms: "gifted*," "neuroimaging," "encephalogra*/ EEG," "event-related potential/ERP," "computerized tomography/CT," "magnetic resonance imaging/MRI," "diffusion tensor imaging/DTI," and "positron emission tomography/ PET." All relevant articles obtained were reviewed and synthesized.

\section{Data Analysis}

Studies obtained in the literature search were organized by neuroimaging method to compare outcomes. Inclusion criteria for sample participants and giftedness identification procedures were considered in comparisons of the results.

\section{Conclusions}

Based on the synthesis of the available literature, conference attendees will gain an understanding of neuroimaging techniques that may help to identify students in need of gifted education services. Given the changing demographics of the USA and the need for identification measures with less probability of ethnic bias, recent advances in neuroimaging should be of interest to pediatric neuropsychologists as potential alternative or supplemental methods for gifted identification.

Author: Lauren E. Moss, M.A. (lemoss@bsu.edu)

Address: 4058 W. 115th St., Apt. 304, Chicago, IL 60655

Office Phone: 708-424-2000 Ext. 2910

Office Fax: Not Applicable
Affiliations: Ball State University; INSPiRE Neuropsychology Clinic, Community High School District 218, Oak Lawn, IL

\section{The Development of Executive Functioning in Bilingual Individuals}

\section{Objectives}

Participants will gain an understanding of how executive functioning skills develop in bilingual individuals; be able to identify differences in executive functioning in different linguistic populations; and learn implications for pediatric neuropsychologists.

\section{Data Selection}

A review of the literature was conducted using Google Scholar and the Ball State University OneSearch databases. Results were limited to include articles from peer-reviewed journals published in the last 10 years. The following search terms were utilized: "bilinguals," "bilingualism," "executive functioning," and "executive functioning development."

\section{Data Analysis}

Data were analyzed using the "preview, question, read, and summarize" approach. Research indicates executive functioning and verbal ability differences exist in bilingual individuals compared to monolinguals, and these differences persist across age groups. Support for an executive functioning advantage in bilingual individuals has been demonstrated in children as young as three. When controlling for verbal ability and demographic variables, bilingual children have demonstrated stronger executive functioning skills when compared to monolingual children and English language learners. In addition to this advantage, a verbal ability deficit when compared to monolingual individuals has been observed in bilingual children. This pattern of performance appears to be maintained throughout adulthood, with research indicating adult bilingual individuals perform better on executive control tasks but worse on lexical retrieval tasks when compared to monolingual adults, even after controlling for age and working memory abilities.

\section{Conclusions}

Results suggest bilingual individuals have additional linguistic barriers to overcome, but their ability to manage two linguistic systems may enhance their performance on executive functioning tasks compared to monolingual individuals. This highlights the importance of conducting comprehensive evaluations when working with bilingual children to better understand their strengths and weaknesses. Future research should emphasize developing strategies that can utilize bilinguals' executive functioning abilities to aid in developing stronger verbal abilities. 
Authors: Deborah K. Kundert, Ph.D. ${ }^{1}$ (dkundert@albany.edu), Julie K. Lynch, Ph.D. ${ }^{2}$ (jklpsy@aol.com), and Robert J. McCaffrey, Ph.D. ${ }^{3}$ (rjmccaffrey@aol.com)

${ }^{1}$ Address: 233 Education, 1400 Washington Ave., Albany, NY 12222

Phone: 518-442-5072

Fax: 518-442-4953

Affiliation: University at Albany

${ }^{123}$ Address: PO Box 1522, Guilderland, NY 12084

Phone: 518-464-5060

Fax: 518-464-5023

Affiliation: Albany Neuropsychological Associates

\section{Integrating Neuropsychological Data Using the Cross Battery Assessment Approach}

\section{Objectives}

This poster will demonstrate the use of the cross battery assessment approach for integrating neuropsychological data in case conceptualization.

\section{Discussion}

Comprehensive neurospsychological evaluations use many assessment techniques resulting in numerous scores. The challenge for clinicians is to analyze and synthesize these results to accurately describe the child, present the results in a clear, organized manner, offer data-supported conclusions, and provide recommendations for interventions.

Often, results are examined and presented test-by-test (e.g., Lichtenberger et al. 2004). Often test names do not accurately indicate the actual domain that is assessed. Additionally, this approach fails to integrate test results, thus placing the burden on the reader to try to interpret the results.

To go beyond the simple descriptive presentation of test data and conclusions, it is now recommended that clinicians integrate information across sources by the domain or skill assessed (e.g., Donders 2016). One method to use in this process is the cross battery assessment approach (XBA; Flanagan et al. 2013). Based on the Cattell-Horn-Carroll theory, XBA allows clinicians to make "systematic, reliable, and theorybased interpretations of cognitive and ... neuropsychological batteries to gain a more psychometrically defensible and complete understanding of an individual's pattern of strengths and weaknesses" (Flanagan et al. 2007, p. 1).

To illustrate the XBA approach, serial neuropsychological evaluation data from a male patient will be categorized, interpreted, and presented in comparison to the traditional test-by-test approach to highlight the ease and accuracy of the resulting case conceptualization. The XBA synthesis of data leads to a report that is focused on the individual child rather than the tests used.

\section{Conclusions}

This presentation provided an example of the use of the theoretically and empirically based XBA to organizing neuropsychological testing data that leads to integrated case conceptualization.

Authors: Noah Sideman MS MEd CBIS ${ }^{1}$ (noahsi@pcom.edu), Sarah Allen $\mathrm{PhD}^{2}$ (sarahll@pcom.edu), Hasan Ayaz $\mathrm{PhD}^{3}$ (hasan.ayaz@drexel.edu), Christine Hammond $\mathrm{MS}^{4}$ (christineh@pcom.edu), Amanda Sargent MS ${ }^{5}$ (as3625@drexel.edu), Lei Wang MS ${ }^{5}$ (lw474@drexel.edu), Jamie Slonim ${ }^{6}$, Prithi Narayan $\mathrm{MD}^{7}$, Denah Appelt $\mathrm{PhD}^{8}$, Brian Balin $\mathrm{PhD}^{8}$ (brianba@pcom.edu)

${ }^{1}$ Address: 725 Tasker St., Philadelphia, 19148

Phone: 215-370-5929

Affiliation: Laboratory Assistant/Graduate Student at the Philadelphia College of Osteopathic Medicine

${ }^{2}$ Address: 4170 City Avenue, Philadelphia, PA 19131

Phone: 215-871-6100

Affiliation: Assistant Professor, Philadelphia College of Osteopathic Medicine, Psychology Department

${ }^{3}$ Address: 3508 Market Street, Monell Suite 101, Philadelphia, PA 19104

Phone: 215-895-2215

Affiliation: Assistant Research Professor, Drexel University, School of Biomedical Engineering, Science \& Health Systems

${ }^{4}$ Address: 4170 City Avenue, Philadelphia, PA 19131

Phone: 215-871-6100

Affiliation: Research Coordinator of Biomedical Sciences, Philadelphia College of Osteopathic Medicine, Department of Bio-Medical Sciences

${ }^{5}$ Address: 3508 Market Street, Monell Suite 101, Philadelphia, PA 19104

Phone: $215-895-2215$

Affiliation: Graduate Student, Drexel University, School of Biomedical Engineering, Science \& Health Systems

${ }^{6}$ Address: Montgomery County Intermediate Unit, Norristown, PA 19403

Affiliation: School Psychologist/PCOM Doctoral Student

${ }^{7}$ Address: 160 East Erie Avenue, Philadelphia, PA 19134 Affiliation: Chief of Neurosurgery, St. Christopher's Hospital for Children, Section of Neurosurgery

${ }^{8}$ Address: 4170 City Avenue, Philadelphia, PA 19131

Phone: 215-871-6100 
Affiliation: Professor of Biomedical Sciences, Philadelphia College of Osteopathic Medicine, Department of BioMedical Sciences

\section{Concussions in Adolescents: a Bio-Behavioral Profile}

\section{Objectives}

Due to findings illuminated in professional sports regarding concussions (Omalu et. al. 2005, 2006; Bales et. al. 2013; Turner et. al. 2014) through documentaries and recent main stream films, concussions in pediatrics and adolescents have become an increased concern for healthcare professionals and families alike. While current research has begun to illustrate the impact of concussions in this population, more investigation is needed to ascertain the broader concussion profile across various evaluative measures. The current IRB-approved pilot study aimed to fill this need by profiling concussions in adolescents across several behavioral and biological domains.

\section{Methods}

Adolescents $(n=3)$ ages $14-17$ within 4 weeks of concussion (diagnosed according to the International Consensus Conference definition) were compared to similar control subjects $(n=3)$. All subjects met inclusion criteria specified in the IRB protocol. Total participation time was approximately $1 \mathrm{~h}$, and all procedures were minimally invasive. Groups were compared across neuropsychological, emotional, neuroimaging, molecular biological (miRNA), olfactory, and symptom reporting dimensions.

\section{Results}

A preliminary analysis of findings revealed differences in sleep duration between groups as well as differences on emotional rating scale measures. Initial neuropsychological findings revealed mostly comparative performances overall with indications of increased reaction times in the concussed group. Smell test results also revealed mostly comparative performances between groups. Findings from miRNA analysis indicated differences in the gene expression of miRNA between groups.

\section{Conclusions}

This pilot study provides a preliminary bio-behavioral profile of concussions in adolescents. Results can help to highlight the sensitivity of evaluative tools that can be utilized in the diagnosis and treatment of concussions in adolescents 2-4 weeks post-injury. Findings can additionally assist in the future development of multimodal concussion diagnostic practices as well as identify bio-behavioral markers that can be tracked to support the rehabilitation process.
Brenda Eagan Brown MEd CBIS ${ }^{1}$ (eaganbrown@biapa.org) and Noah Sideman MS MEd² (noahsi@pcom.edu)

${ }^{1}$ Address: 950 Walnut Bottom Road, Suite 15-229, Carlisle, PA 17015

Phone: 724.944 .6542

Fax: 717-692-5567

Affiliations: BrainSTEPS Program Coordinator, Brain Injury School Re-Entry Consulting Program, Brain Injury Association of PA

${ }^{2}$ Address: 725 Tasker St., Philadelphia, 19148

Phone: 215-370-5929

Affiliation: Graduate Student, Philadelphia College of Osteopathic Medicine

\section{Pennsylvania's Statewide Return to Learn Concussion Management Teams}

\section{Objectives}

It is estimated that over 20,000 students in Pennsylvania (PA) sustain concussions each year. Many return to school with lingering effects that impact classroom performance. Return to Learn Concussion Management Teams (CMT) were created in PA to build school's capacity to manage students' academic progress and physical symptoms in the initial weeks post-concussion.

\section{Method}

The PA Department of Education contacted all schools to identify academic and symptom monitors to serve on CMT. These teams completed an online training developed by BrainSTEPS and hosted by the PA Department of Health. Upon completion of the training, CMT received a monitoring toolkit to track student academics and physical symptoms for the initial weeks post-concussion. A subset of CMT voluntarily submitted deidentified student concussion data which is reviewed in this presentation.

\section{Results}

Over 3500 student concussions were supported by CMT in PA over a 2-year period (September 2012-December 2014), and $880 \mathrm{CMT}$ were created across the state. The gender of concussed students supported by CMT was near equal (52\% male vs. $48 \%$ female). Most concussions occurred in the 9th-11th grades and at ages 15-17. Most students returned to school within $0.5-4.5$ days $(46 \%)$ and more than half $(52 \%)$ were 1 week symptom free by 3 weeks. Furthermore, nearly half of students (49\%) returned to academic baseline by 2 weeks post-injury and $76 \%$ at 4 weeks. Only $1 \%$ of students spent longer than 20.5 days out of school. 


\section{Conclusions}

Findings highlight the importance of monitoring both academics and physical symptoms and shows the importance of statewide concussion management programs for pediatrics. Furthermore, as a result of CMT, students with unresolved symptoms and perhaps more severe TBI were more readily identified and provided with more intensive support through PA's BrainSTEPS program.

Authors: Theodore Wasserman PhD, ABPP, ABPdN (drtedwasserman@gmail.com), Angela M. Mion, M.A. (ammion@bsu.edu)

Address: 2000 W. University Ave., Teachers College 505, Muncie, IN 47306

Office Phone: 765-285-8500

Office Fax: 765-285-3653

Affiliation: Ball State University

\section{Long-Term Memory Loss Secondary to White Matter Attenuation: a Case Study}

\section{Objectives}

Many studies demonstrate that early cognitive deficits in cases of mTBI are largely resolved within about 3 months post-injury (Carrol et al. 2004; Macciocchi et al. 1996). There is increasing evidence that suggests that for a subset of individuals with TBI, the course post-injury does not follow what has been traditionally anticipated. Bigler (2012) hypothesized that part of the explanation for these progressive changes, particularly in white matter, far beyond the stable lesion phase, may be the result of TBI-initiated chronic neuroinflammatory processes. The youth, a 12-year-old male, sustained a traumatic brain injury when a thrown baseball traveling approximately 45 miles per hour hit him in the left frontal temporal region causing a left temporal lobe displaced skull fracture with an underlying hemorrhage.

\section{Methods}

Six weeks post-injury, he was administered the Wide Range Assessment Test of Memory and Learning, Second Edition and the California Verbal Learning Test, Children's Version. Two years later, he was administered the Children's Memory and the Wechsler Memory Scales, Fourth Edition. Three years post-injury, diffusion tensor imaging was conducted on the youth.

\section{Results}

Results of testing after 6 weeks were within normal limits. Results of testing after 2 years showed significant memory deficits. Diffusor imaging demonstrated a pattern and distribution consistent with post-traumatic loss of white matter integrity.

\section{Conclusions}

It is possible that the white matter damage had been initiated and continued to worsen over time, for a period longer than what is customarily assumed. It was not that the tests were not sufficiently sensitive to detect the behavioral sequelae of the injury, it was that the sequelae had not become fully articulated and would not do so until the while matter attenuation attained a critical level.

\section{References}

Bigler, E. (2012). Neuropathology of mild traumatic brain injury: relationship to neuroimaging findings. Brain Injury and Behavior, 6(2), 108-136. doi: 10.1007/ s11682-011-9145-0

Carrol, L., Cassidy, J., Peloso, P., Borg, J., von Holst, H., Holm, L.,...Pepin, M. (2004). Prognosis for mild traumatic brain injury: results of the WHO Collaborating Centre Task Force on Mild Traumatic Brain Injury. Journal of Rehabilitative Medicine; Suppl. 43, 84-105. doi 10.1080/ 16501960410023859.

Macciocchi, S., Barth, J., Alves, W., Rimel, R., \& Jane, J. (1996). Neuropsychological functioning and recovery after mild head injury in college athletes. Neurosurgery, 39, 510-514.

Authors: Donald T. Vercellini M.A.' (dvercellini@alliant.edu), Brittany A. Cunningham M.A. ${ }^{2}$ (bcunningham@alliant.edu), Paul C. Lebby Ph.D. ${ }^{3}$ (plebby@ Valleychildrens.org), and Merle Canfield Ph.D. ${ }^{4}$ (mcanfield@alliant.edu)

${ }^{1}$ Phone: 559-977-1307

Affiliation: California School of Professional Psychology (3248 Bundy Ave. Clovis, CA 93611), Alliant International University, Fresno (5130 E Clinton Way, Fresno, CA 93727)

${ }^{2}$ Address: 1234 E. Champlain Dr. \#208 Fresno, CA 00000 Phone: 530-845-1429

Affiliation: California School of Professional Psychology, Alliant International University, Fresno (5130 E Clinton Way, Fresno, CA 93727)

${ }^{3}$ Address: 9300 Valley Children's Place, Madera, CA 93636 Affiliation: Valley Children's Hospital, Department of Clinical Neuropsychology

${ }^{4}$ Address: 5130 E Clinton Way, Fresno, CA 93727 Phone: 917-881-1398 
Affiliation: California School of Professional Psychology, Alliant International University, Fresno

\section{Structural Model Analysis of the Lebby-Asbell Neurocognitive Screening Exams}

\section{Objectives}

Neuropathological disorders (NPD) and traumatic brain injuries (TBI) are a leading cause of child and adolescent morbidity and mortality. Their frequency in acute care settings creates a pressing need for quick and efficient assessment of neurocognitive integrity. The Lebby-Asbell Neurocognitive Screening Examination for Children (LANSE-C) and Lebby-Asbell Neurocognitive Screening Examination for Adolescents (LANSE-A) meet this need. Previous multivariate analyses of these instruments demonstrated excellent reliability, validity, specificity, sensitivity, and positive predictive power. However, because the LANSE-C/A are currently the only published neurocognitive screening instruments designed specifically for children and adolescents, higher order statistical analysis is justified. We hypothesized that (1) The LANSE-C/A's 14 subtests would load in a manner consistent with the test's theoretical functional domains. (2) The analysis would support earlier findings of validity and reliability. (3) The instrument would differentiate between NPD and TBI.

\section{Methods}

The LANSE-C/A was administered to children and adolescents presenting with (NPD) and (TBI) at Valley Children's Hospital in Madera, California $(N=129)$. The hospital's 45, 000 -square-mile service area covers most of California's central San Joaquin Valley, housing one of the most diverse populations in the USA.

\section{Results}

Structural model analysis affirmed the LANSE-C/A measurement model while accounting for $20 \%$ of the variance between NPD and TBI $(R=.44)$. Statistically based factor loadings were consistent with the clinically derived model.

\section{Conclusions}

The LANSE-C/A's clinically derived domains and subtest construct validity were supported confirming the internal structure of the LANSE-C/A's and affirming the rationale underlying the instrument design. The results confirmed earlier findings of validity and reliability and the clinical utility of the LANSE-C/A as effective assessment instruments, with generalizability to clinical case conceptualization and treatment planning in pediatric acute care settings.

Authors: Arthur MacNeill Horton, Jr., Ed.D, ABPP (CL), ABN, ABPP (BP) (drmachorton@gmail.com) and Cecil R. Reynolds, PhD. ABN, ABPdN (crrh@earthlink.net)
Address: 5903 Lone Oak Drive, Bethesda, MD 20814

Office Phone: 3012193054

Office Fax: 3015303417

Affiliation: Neuropsychology Section, Psych Associates of Maryland LLC

\section{Teen Brain and the Death Penalty}

\section{Objectives}

Pediatric neuropsychological assessment methods are the standard of both practice and research in examining the functional capacity of the child and adolescent brain as it relates to behavior and how behavior changes as the child and adolescent brain develops (normally and abnormally) or is damaged or otherwise compromised. Clinical neuropsychological testing results are well accepted in settings such as medical, educational, and legal arenas. Neuropsychological testing in clinical practice typically encompasses the assessment of multiple neuropsychological domains, but the most important of all neuropsychological domains has been executive functioning. The neuropsychological construct of executive functioning relates to human adaptive abilities, problem-solving, and the development as well as inhibition of behavioral plans. This poster will review data regarding the importance of considering pediatric neuropsychology findings regarding executive functioning in making decisions regarding the death penalty in the USA.

\section{Conclusions}

Current knowledge of the functional trajectory of executive functioning abilities' development should promote a reconsideration of the age of eligibility for the sentence of death in capital cases (which is now 18 years) given the continued development of executive functioning and the neuroanatomical regions of the human brain involved (e.g., frontal lobes, etc.). In addition, current knowledge of the functional trajectory of executive functioning ability development should promote a reconsideration of issues related to judgments of legal concepts of mitigation and future dangerousness. Executive functions are controlled by the prefrontal regions of the brain and the neural pathways allowing this region of the brain to communicate well with other brain regions, thus regulating our behavior. These are the regions of the brain most heavily involved in evaluating risk, controlling behavior, and decision-making, preventing us from behaving when behavior is inappropriate or damaging.

Authors: Arthur MacNeill Horton, Jr., Ed.D, ABPP (CL), ABN, ABPP (BP) (drmachorton@gmail.com) and Cecil R. Reynolds, PhD, ABN, ABPdN (crrh@earthlink.net)

Address: 5903 Lone Oak Drive, Bethesda, MD 20814

Office Phone: 3012193054

Office Fax: 3015303417 
Affiliation: Neuropsychology Section, Psych Associates of Maryland LLC

Pediatric Neuropsychology Assessment with the Test of Verbal Conceptualization and Fluency

\section{Objectives}

This poster provides an introduction to the Test of Verbal Conceptualization and Fluency (TVCF), a new measure for the pediatric assessment of executive functions. The TVCF is a standardized set of four subtests with a total administration time of 25-30 min for most individuals. The test is designed to measure multiple aspects of executive functions, through the use of several different forms of tasks commonly used by pediatric neuropsychologists to assess executive functioning. Executive functions can be contrasted with knowledge relates to retention of an organized set of facts and executive functions deal with problem-solving and performing adaptive actions. Knowledge relates to the passive retention of information, while executive functioning involves motor outputs that are adaptive to external demands.

\section{Conclusions}

A crucial insight is that while executive functioning impairment can be caused by frontal lobe brain injury, executive functioning impairment can also because by impairments in brain areas other than the frontal lobes Executive functioning, although it can be related to impairment in the frontal lobes, is not synonymous with frontal lobe impairment. The frontal lobes are a neuroanatomical area of human brain, while executive functioning is a neuropsychological concept/domain similar to attention and memory. Traditional clinical neuropsychological measures of executive functioning that were selected for inclusion and modification in the TVCF include card sorting tasks, category and letter retrieval tasks, and "trail making" tasks. The aim of the TVCF is provide a standardized assessment of executive functioning to better conceptualize and evaluate this important area of mental functioning and adaptive functioning. In addition, the hope is the standardized assessment and the use of co-normed tasks to allow more accurate interpretation of executive functioning abilities. 